\title{
Inclusive gluon production in high energy onium-onium scattering
}

\author{
Yuri V. Kovchegov* \\ Department of Physics, The Ohio State University, Columbus, Ohio 43210, USA
}

(Received 2 September 2005; published 11 November 2005)

\begin{abstract}
We calculate the inclusive single-gluon production cross section in high energy onium-onium scattering including pomeron loop effects. The resulting inclusive cross section is given by the $k_{T}$-factorization formula with one of the unintegrated gluon distribution functions depending on the total onium-onium scattering cross section, which includes all pomeron loops and has to be found independently. We discuss the limits of applicability of our result and argue that they are given by the limits of applicability of the pomeron loop resummation approach. Since the obtained $k_{T}$-factorization formula is infrared divergent we conclude that, in order to consistently calculate the (infrared-finite) gluon production cross section in onium-onium scattering, one has to include corrections going beyond the pomeron loop approximation.
\end{abstract}

DOI: 10.1103/PhysRevD.72.094009

PACS numbers: $13.85 . \mathrm{Ni}, 13.60 . \mathrm{Hb}, 13.85 . \mathrm{Hd}$

\section{INTRODUCTION}

Recently there has been a lot of renewed interest in resummation of the so-called "pomeron loop" diagrams for high energy scattering [1-8]. These diagrams are particularly important for the calculation of the cross section for high energy scattering of two quarkonia. It has been argued that the growth of the onium-onium total scattering cross section with energy due to the Balitsky-FadinKuraev-Lipatov (BFKL) evolution [9] is unitarized (at fixed impact parameter) by the multiple hard pomeron exchanges realized by the pomeron loop diagrams [1014]. Such unitarization is qualitatively different from the unitarization of the total scattering cross section in deep inelastic scattering (DIS) on a nucleus [15-20]: in the onium-onium scattering case one considers a collision of two small and dilute objects, whereas in the case of DIS one of the scatterers is a large and dense nucleus.

The contribution of multiple pomeron exchanges to the total onium-onium scattering cross section has been studied within the framework of Mueller's dipole model [10] both analytically by Mueller and Patel [11] and Navelet and Peschanski [13] and numerically by Salam in [14]. The approach of $[11,13,14]$ has recently been reformulated in the language of the color glass condensate by Iancu and Mueller in [1]. The main idea behind the dipole model approach to onium-onium scattering is reviewed in Sec. II. When viewed in the center-of-mass frame, each of the colliding onia develops a dipole wave function. The dipoles in the light-cone wave functions of the onia interact pairwise by two-gluon exchanges $[1,11]$. This process is equivalent to resumming the pomeron loop diagrams in the traditional Feynman diagram language and is illustrated below in Fig. 2.

Our goal in this paper is to calculate the single-gluon inclusive production cross section in the onium-onium scattering described in the framework of Mueller's dipole model $[1,11]$. This is an interesting observable one could

*Email address: yuri@mps.ohio-state.edu study in $\gamma^{*} \gamma^{*}$ scattering at the future linear colliders. The calculation turns out to be analogous to the case of gluon production in DIS considered in [21] and is outlined in Sec. III. Using the real-virtual cancellations of some of the final state interactions observed in [22] we arrive at the expression for the inclusive cross section of the singlegluon production given by Eq. (23). As one can also see from a different form of Eq. (23) given by Eq. (29), the resulting gluon production cross section adheres to Abramovsky-Gribov-Kancheli (AGK) cutting rules [23] and is given by the $k_{T}$-factorization expression [24], similar to the results of $[21,25,26]$ for DIS and to [27]. The $k_{T}$ factorization appears here because of the fact that, due to the nature of the approximation of $[1,11]$, one of the onia wave functions (the one closer to the produced gluon in rapidity) appears to be unsaturated, i.e., has no saturation effects in it and is described simply by the linear BFKL evolution. This makes the problem effectively identical to the case of DIS or $p A$ collisions.

The $k_{T}$-factorization formula for the gluon production cross section is known to diverge as $\sim 1 / k_{T}^{2}$ in the infrared [28] (here $k_{T}$ is the transverse momentum of the produced gluon). This divergence is believed to be curable if one includes saturation effects in both of the onia wave functions, which would lead to a breakdown of the $k_{T}$-factorization expression but would yield an infraredfinite production cross section (a similar observation appears to hold for gluon production in nucleus-nucleus collisions [29-31]). For the onium-onium scattering, saturation in both of the onia wave functions can be included by resumming all pomeron loop diagrams with the loops spanning less than a half of the total rapidity interval. However, as we discuss in Sec. IV, when rapidity is high enough for such "small" pomeron loops to become important, other corrections to the pomeron loops approach become equally important as well. Some of these nonpomeron loop corrections have been discussed in [6] to explain the discrepancy between the results of $[2,5]$. In the traditional Feynman diagram language these corrections can be associated with higher order terms in the impact 
factors, zero-rapidity size pomeron loops and/or with iterations of next-to-leader order (NLO) and next-to-next-toleading order (NNLO) BFKL kernels in the LO BFKL ladder. Therefore, we arrive at a somewhat unsettling conclusion that in order to obtain an infrared-finite gluon production cross section in onium-onium scattering in a consistent way one may have to go beyond the pomeron loop approximation to high energy scattering.

\section{ONIUM-ONIUM SCATTERING AT INTERMEDIATE HIGH ENERGY}

Let us consider high energy scattering of two quarkonia in the approximation put forward in $[1,11,12]$. The notations we use are explained in Fig. 1. The onia have transverse sizes $\underline{x}_{01}=\underline{x}_{0}-\underline{x}_{1}$ and $\underline{x}_{0^{\prime} 1^{\prime}}=\underline{x}_{0^{\prime}}-\underline{x}_{1^{\prime}}$ with $\underline{x}_{0}, \underline{x}_{1}, \underline{x}_{0^{\prime}}, \underline{x}_{1^{\prime}}$ the transverse coordinates of quarks and antiquarks in both onia. The impact parameter of the collision is $\underline{B}$ and the rapidity interval between the onia is $Y$.

The dipole model description of high energy oniumonium scattering $[1,11,12]$ is illustrated in Fig. 2(a). In the center-of-mass frame, each of the onia develops a system of color dipoles in its light-cone wave function before the collisions. At the time of the collision [denoted by the dash-dotted line in Fig. 2(a)] the dipoles in both onia interact with each other pairwise by two-gluon exchanges.

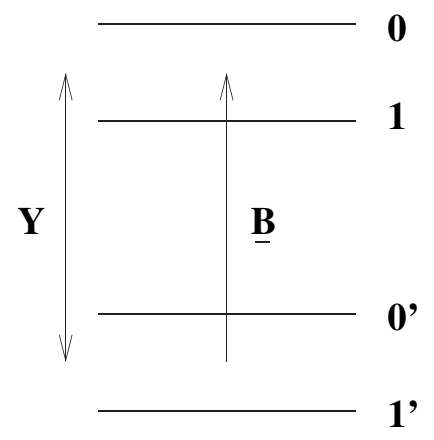

FIG. 1. Onium-onium scattering as considered in the text.

In Fig. 2(a) we show the case when three pairs of dipoles interact with each other. This corresponds to a threepomeron exchange in the traditional Feynman diagram language, which is illustrated in Fig. 2(b). In the dipole model [11,12], the BFKL evolution [9], which is included in the ladder diagrams in Fig. 2(b), comes in through the dipole evolution in the light-cone wave functions of the onia, which leads to a generation of the interacting dipoles.

The dipole wave function of an onium is described by the generating functional for dipoles $Z\left(\underline{x}_{01}, \underline{b}, Y, u\right)$, which obeys the following evolution equation $[10,12]$ :

$$
\begin{aligned}
Z\left(\underline{x}_{01}, \underline{b}, Y, u\right)= & u\left(\underline{x}_{01}, \underline{b}\right) e^{-2 \bar{\alpha}_{s} \ln \left(x_{01} / \rho\right) Y}+\frac{\bar{\alpha}_{s}}{2 \pi} \int_{0}^{Y} d y e^{-2 \bar{\alpha}_{s} \ln \left(x_{01} / \rho\right)(Y-y)} \\
& \times \int_{\rho} d^{2} x_{2} \frac{x_{01}^{2}}{x_{02}^{2} x_{12}^{2}} Z\left(\underline{x}_{02}, \underline{b}+\frac{1}{2} \underline{x}_{12}, y, u\right) Z\left(\underline{x}_{12}, \underline{b}-\frac{1}{2} \underline{x}_{20}, y, u\right),
\end{aligned}
$$

with

$$
\bar{\alpha}_{s} \equiv \frac{\alpha_{s} N_{c}}{\pi}
$$

and $u\left(\underline{x}_{01}, \underline{b}\right)$ a function describing a dipole of transverse size $\underline{x}_{01}$ at impact parameter $\underline{b}$ [12].

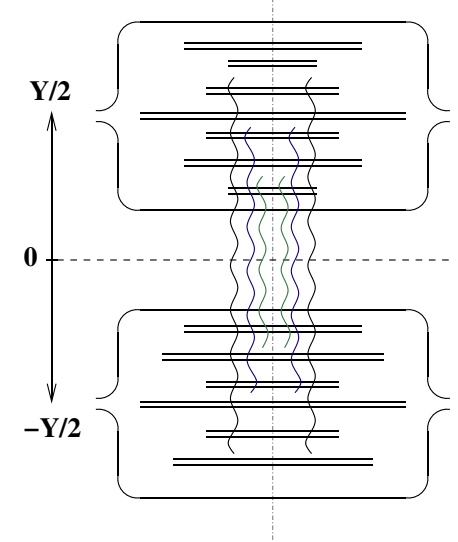

A

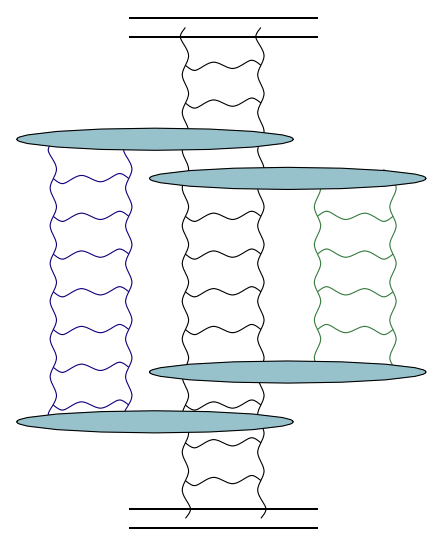

B

FIG. 2 (color online). Three-pomeron exchange diagram for onium-onium scattering in (a) the dipole model and in (b) the Feynman diagram language. The double lines in (a) denote gluons in the large- $N_{c}$ limit. The ovals in (b) denote triple pomeron vertices. 
Using $Z\left(\underline{x}_{01}, \underline{b}, Y, u\right)$ one can define the density of $k$ dipoles in the onium wave function by [11]

$$
\begin{aligned}
& n_{k}\left(\underline{x}_{01}, \underline{b}, Y ; \underline{r}_{1}, \underline{b}_{1} ; \ldots ; \underline{r}_{k}, \underline{b}_{k}\right) \\
& \quad=\left.\frac{1}{k !} \frac{\delta^{k} Z\left(\underline{x}_{01}, \underline{b}, Y, u\right)}{\delta u\left(\underline{r}_{1}, \underline{b}_{1}\right) \cdots \delta u\left(\underline{r}_{k}, \underline{b}_{k}\right)}\right|_{u=1} .
\end{aligned}
$$

If normalized appropriately, $n_{k}$ would have the meaning of inclusive probability of finding $k$ dipoles of given sizes $\underline{r}_{i}$ 's at impact parameters $\underline{b}_{i}$ 's along with any number of extra dipoles. Note that $n_{k}$, as defined by Eq. (3), is normalized to give a density of $k$ dipoles rather than probability.

Let us denote the forward amplitude of dipole-dipole scattering mediated by a two-gluon exchange as

$$
-d\left(\underline{r}, \underline{r}^{\prime}, \underline{b}\right)=\alpha_{s}^{2} \frac{C_{F}}{N_{c}} \ln ^{2}\left(\frac{\left|\underline{b}+(1 / 2) \underline{r}+(1 / 2) \underline{r}^{\prime}\right|\left|\underline{b}-(1 / 2) \underline{r}-(1 / 2) \underline{r}^{\prime}\right|}{\left|\underline{b}+(1 / 2) \underline{r}-(1 / 2) \underline{r}^{\prime}\right|\left|\underline{b}-(1 / 2) \underline{r}+(1 / 2) \underline{r}^{\prime}\right|}\right) .
$$

Here $\underline{r}$ and $\underline{r}^{\prime}$ are transverse separations of the two dipoles and $\underline{b}$ is their impact parameter.

With the aid of the definition from Eq. (4) we can write the forward scattering amplitude for onium-onium scattering, which includes a small- $x$ evolution, as

$$
\begin{aligned}
-D\left(\underline{x}_{01}, \underline{x}_{0^{\prime} 1^{\prime}}, \underline{B}, Y\right)= & -1+\sum_{k=1}^{\infty} k ! \int d^{2} r_{1} d^{2} b_{1} \cdots d^{2} r_{k} d^{2} b_{k} d^{2} r_{1}^{\prime} d^{2} b_{1}^{\prime} \cdots d^{2} r_{k}^{\prime} d^{2} b_{k}^{\prime} n_{k}\left(\underline{x}_{01}, \underline{B}, Y / 2 ; \underline{r}_{1}, \underline{b}_{1} ; \cdots ; \underline{r}_{k}, \underline{b}_{k}\right) \\
& \times n_{k}\left(\underline{x}_{0^{\prime} 1^{\prime}}, \underline{0},-Y / 2 ; \underline{r}_{1}^{\prime}, \underline{b}_{1}^{\prime} ; \cdots ; \underline{r}_{k}^{\prime}, \underline{b}_{k}^{\prime}\right)(-1)^{k} d\left(\underline{r}_{1}, \underline{r}_{1}^{\prime}, \underline{b}_{1}-\underline{b}_{1}^{\prime}\right) \cdots d\left(\underline{r}_{k}, \underline{r}_{k}^{\prime}, \underline{b}_{k}-\underline{b}_{k}^{\prime}\right),
\end{aligned}
$$

where we chose the impact parameters of dipoles 01 and $0^{\prime} 1^{\prime}$ to be $\underline{B}$ and zero correspondingly. The notations of Eq. (5) are explained in Fig. 1.

Using Eq. (3) in Eq. (5) we rewrite it as

$$
\begin{aligned}
-D\left(\underline{x}_{01}, \underline{x}_{0^{\prime} 1^{\prime}}, \underline{B}, Y\right)= & -1+\left.\sum_{k=1}^{\infty} \frac{1}{k !} \int d^{2} r_{1} d^{2} b_{1} \cdots d^{2} r_{k} d^{2} b_{k} d^{2} r_{1}^{\prime} d^{2} b_{1}^{\prime} \cdots d^{2} r_{k}^{\prime} d^{2} b_{k}^{\prime} \frac{\delta^{k} Z\left(\underline{x}_{01}, \underline{B}, Y / 2, u\right)}{\delta u\left(\underline{r}_{1}, \underline{b}_{1}\right) \cdots \delta u\left(\underline{r}_{k}, \underline{b}_{k}\right)}\right|_{u=1} \\
& \times\left.\frac{\delta^{k} Z\left(\underline{x}_{0^{\prime} 1^{\prime}}, \underline{0},-Y / 2, v\right)}{\delta v\left(\underline{r}_{1}^{\prime}, \underline{b}_{1}^{\prime}\right) \cdots \delta v\left(\underline{r}_{k}^{\prime}, \underline{b}_{k}^{\prime}\right)}\right|_{v=1}(-1)^{k} d\left(\underline{r}_{1}, \underline{r}_{1}^{\prime}, \underline{b}_{1}-\underline{b}_{1}^{\prime}\right) \cdots d\left(\underline{r}_{k}, \underline{r}_{k}^{\prime}, \underline{b}_{k}-\underline{b}_{k}^{\prime}\right),
\end{aligned}
$$

where we relabeled functions $u$ by $v$ for the dipole $0^{\prime} 1^{\prime}$. If one could solve Eq. (1) for an arbitrary function $u$, then the solution could be used in Eq. (6) to construct the forward amplitude of onium-onium scattering. Unfortunately no general analytical solution of Eq. (1) for an arbitrary function $u$ is known.

Equation (6) can be recast in a more compact form:

$$
\begin{aligned}
D\left(\underline{x}_{01}, \underline{x}_{0^{\prime} 1^{\prime}}, \underline{B}, Y\right)= & -\left\{\operatorname { e x p } \left[-\int d^{2} r d^{2} b d^{2} r^{\prime} d^{2} b^{\prime} d\left(\underline{r}, \underline{r}^{\prime}, \underline{b}-\underline{b}^{\prime}\right)\right.\right. \\
& \left.\left.\times \frac{\delta^{2}}{\delta u(\underline{r}, \underline{b}) \delta v\left(\underline{r}^{\prime}, \underline{b}^{\prime}\right)}\right] Z\left(\underline{x}_{01}, \underline{B}, Y / 2, u\right) Z\left(\underline{x}_{0^{\prime} 1^{\prime}}, \underline{0},-Y / 2, v\right)\right\}\left.\right|_{u=1, v=1} .
\end{aligned}
$$

This is our final expression for the onium-onium forward scattering amplitude here. One could also try to rewrite Eq. (7) in terms of a functional integral over functions $u$ and $v$ : however, such an integral representation appears to require a non-Gaussian weight functional, which is probably related to the non-Gaussian noise discussed in [2] within the context of adding pomeron loop corrections to Jalilian-Marian-Iancu-McLerran-Weigert-LeonidovKovner evolution equations $[19,20]$.

The question of whether $D\left(\underline{x}_{01}, \underline{x}_{0^{\prime} 1^{\prime}}, \underline{B}, Y\right)$ gives a unitary total onium-onium scattering cross section has been addressed numerically by Salam in [14]. It was demonstrated by Monte Carlo simulations in [14] that $D\left(\underline{x}_{01}, \underline{x}_{0^{\prime} 1^{\prime}}, \underline{B}, Y\right)$ is unitary at a fixed impact parameter $\underline{b}$. To simplify the analysis of $D\left(\underline{x}_{01}, \underline{x}_{0^{\prime} 1^{\prime}}, \underline{B}, Y\right)$ in Eq. (7) it would be very useful to find an integral/differential equation governing the high energy evolution of $D\left(\underline{x}_{01}, \underline{x}_{0^{\prime} 1^{\prime}}, \underline{B}, Y\right)$, similar to the case of DIS considered in $[17,18]$. However, despite many attempts, no such equation for $D\left(\underline{x}_{01}, \underline{x}_{0^{\prime} 1^{\prime}}, \underline{B}, Y\right)$ has been found at this time.

We illustrate the onset of unitarity in $D$ from Eq. (7) by using a simple toy model, originally proposed by Mueller in [12]. Suppressing the transverse coordinate dependence we write the toy model analog of Eq. (1) as [12]

$$
\frac{d Z}{d Y}=\alpha_{s} Z^{2}-\alpha_{s} Z
$$

with the initial condition given by

$$
Z(Y=0, u)=u .
$$

The solution of the toy evolution equation (8) satisfying the initial condition (9) is [12]

$$
Z(Y, u)=\frac{u}{u+(1-u) e^{\alpha_{s} Y}} .
$$

The toy model analog of Eq. (7) is 
$D(Y)=1-\left.\left\{\exp \left[-d \frac{\delta^{2}}{\delta u \delta v}\right] Z(Y / 2, u) Z(Y / 2, v)\right\}\right|_{u=1, v=1}$,

where $d$ is some positive small number, $0<d<1$, and we put $Y / 2$ instead of $-Y / 2$ in the argument of $Z(Y / 2, v)$ to underline the fact that it is the absolute value of the rapidity interval, and not the rapidity itself, which matters there. Substituting $Z$ from Eq. (10) into Eq. (11), expanding the exponential, and performing the differentiations with respect to $u$ and $v$ yields

$$
D(Y)=-\sum_{n=1}^{\infty} n !(-1)^{n} d^{n} e^{\alpha_{s} Y}\left(1-e^{\alpha_{s} Y / 2}\right)^{2 n-2} .
$$

The series in Eq. (12) is divergent; however, since $d>0$, it is Borel summable. At large rapidities we can approximate $1-e^{\alpha_{s} Y / 2} \approx-e^{\alpha_{s} Y / 2}$ rewriting Eq. (12) as

$$
D(Y) \approx-\sum_{n=1}^{\infty} n !(-1)^{n} d^{n} e^{n \alpha_{s} Y}
$$

which after Borel resummation yields

$$
D(Y) \approx 1-\frac{1}{d e^{\alpha_{s} Y}} \exp \left(\frac{1}{d e^{\alpha_{s} Y}}\right) \Gamma\left(0, \frac{1}{d e^{\alpha_{s} Y}}\right) .
$$

The amplitude $D(Y)$ from Eq. (14) is shown in Fig. 3 as a function of $\alpha_{s} Y$. One can see that at very large $Y$ the black disk limit $(D=1)$ is reached and is never exceeded, i.e., the amplitude is unitary.

It is interesting to note that if one does not make the $1-$ $e^{\alpha_{s} Y / 2} \approx-e^{\alpha_{s} Y / 2}$ approximation and Borel resums the full series in Eq. (12), the resulting amplitude would violate unitarity: as one increases rapidity it would grow, becoming greater than 1 at some large rapidity $Y$, and, after reaching a maximum it would slowly decrease, approaching 1 from above at asymptotically high rapidities. Indeed the pomeron loop approximation of $[1,11]$ consistently resums only the leading terms at high energy, and has no

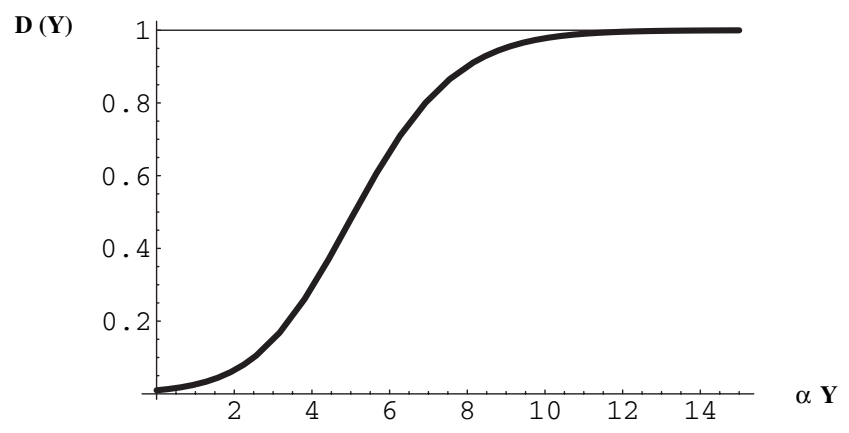

FIG. 3. Onium-onium scattering amplitude $D(Y)$ from the toy model result in Eq. (14) plotted as a function of $\alpha_{s} Y$ for $d=$ 0.01 (thick line). The thin horizontal line denotes the black disk limit. control over the subleading terms, such as 1 that we neglected compared to $e^{\alpha_{s} Y / 2}$. Therefore, such pathological behavior of $D(Y)$ is probably due to the fact that the toy model at hand, along with the approximation of $[1,11]$, does not consistently resum the subleading terms. However, it may also indicate the importance of subleading corrections for onium-onium scattering at very high rapidities. After all, the same toy model would always give a unitary cross section for DIS, where the dipole-nucleus forward scattering amplitude is given by $N=1-Z$ $[17,18]$. We will return to this discussion in Sec. IV.

\section{INCLUSIVE GLUON PRODUCTION}

To calculate a single-gluon production cross section in the approximation to onium-onium scattering developed in $[1,11]$ we start with the lowest order contribution. One of the corresponding diagrams is shown in Fig. 4. The produced gluon is denoted by the cross. The disconnected $s$-channel (horizontal) gluon line in Fig. 4 indicates that the gluon can be emitted off either the quark or the antiquark lines in the onium 01 . The disconnected $t$-channel (vertical) gluon line denotes the sum of the gluon interactions with the emitted gluon, quark, and antiquark in dipole 01 at one end, and with the quark and the antiquark in dipole $0^{\prime} 1^{\prime}$ at the other end.

The dipole model approach to onium-onium scattering [11] was developed in the Coulomb gauge, which is equivalent to the light-cone $A_{+}=0$ gauge for the wave function of the onium moving in the light-cone "+" direction and to the $A_{-}=0$ gauge for the wave function of the onium moving in the light-cone "- " direction. In analyzing the lowest order diagram from Fig. 4 we will work in the $A_{+}=0$ light-cone gauge, where the 01 onium is taken to be going in the " + " direction and the $0^{\prime} 1^{\prime}$ onium is taken to be going in the "-" direction.

The lowest order gluon production cross section is well known [32-34]. Following [21,35] we can write it as (for a review of similar derivations see [36])

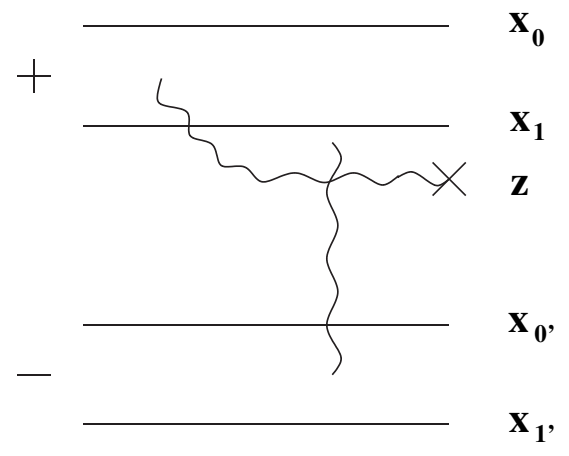

FIG. 4. Lowest order gluon production in onium-onium scattering. 


$$
\begin{aligned}
\frac{d \sigma_{\mathrm{LO}}\left(\underline{x}_{01}, \underline{x}_{0^{\prime} 1^{\prime}}\right)}{d^{2} k d y d^{2} B}= & \frac{\alpha_{s} C_{F}}{\pi^{2}} \frac{1}{(2 \pi)^{2}} \int d^{2} z_{1} d^{2} z_{2} e^{-i \underline{k} \cdot\left(\underline{z}_{1}-\underline{z}_{2}\right)} \sum_{i, j=0}^{1}(-1)^{i+j} \frac{\underline{z}_{1}-\underline{x}_{i}}{\left|\underline{z}_{1}-\underline{x}_{i}\right|^{2}} \cdot \frac{\underline{z}_{2}-\underline{x}_{j}}{\left|\underline{z}_{2}-\underline{x}_{j}\right|^{2}}\left[d_{G q}\left(\underline{z}_{1}-\underline{x}_{j}, \underline{x}_{0^{\prime} 1^{\prime}}, \frac{1}{2}\left(\underline{z}_{1}+\underline{x}_{j}\right)\right)\right. \\
& \left.+d_{G q}\left(\underline{z}_{2}-\underline{x}_{i}, \underline{x}_{0^{\prime} 1^{\prime}}, \frac{1}{2}\left(\underline{z}_{2}+\underline{x}_{i}\right)\right)-d_{G q}\left(\underline{z}_{1}-\underline{z}_{2}, \underline{x}_{0^{\prime} 1^{\prime}}, \frac{1}{2}\left(\underline{z}_{1}+\underline{z}_{2}\right)\right)-d_{G q}\left(\underline{x}_{i}-\underline{x}_{j}, \underline{x}_{0^{\prime} 1^{\prime}}, \frac{1}{2}\left(\underline{x}_{i}+\underline{x}_{j}\right)\right)\right],
\end{aligned}
$$

where

$$
d_{G q}\left(\underline{r}, \underline{r}^{\prime}, \underline{b}\right)=\frac{N_{c}}{C_{F}} d\left(\underline{r}, \underline{r}^{\prime}, \underline{b}\right)=\alpha_{s}^{2} \ln ^{2}\left(\frac{\left|\underline{b}+(1 / 2) \underline{r}+(1 / 2) \underline{r}^{\prime}\right|\left|\underline{b}-(1 / 2) \underline{r}-(1 / 2) \underline{r}^{\prime}\right|}{\left|\underline{b}+(1 / 2) \underline{r}-(1 / 2) \underline{r}^{\prime}\right|\left|\underline{b}-(1 / 2) \underline{r}+(1 / 2) \underline{r}^{\prime}\right|}\right)
$$

is the forward amplitude for the scattering of an adjoint (gluon) dipole of size $\underline{r}$ on a fundamental (quark) dipole of size $\underline{r}^{\prime}$ separated by the impact parameter $\underline{b}$ and mediated by a two-gluon exchange. Again we assume that the impact parameter of the onium $0^{\prime} 1^{\prime}$ is zero. In Eq. (15), $\underline{k}$ and $y$ are the transverse momentum and rapidity of the produced gluon, while $\underline{z}_{1}$ and $\underline{z}_{2}$ are its transverse coordinates, which are different on both sides of the cut $[21,29,35,36]$.

The task now is to include the effects of the small- $x$ evolution taking place in both of the onia's wave functions in Eq. (15). In our approach we will be closely following the case of gluon production in DIS considered previously in [21]. Just like in Fig. 2 we will consider onium-onium scattering in the center-of-mass frame, and, like in [11] we will be working in the Coulomb gauge. We will consider the case in which the produced gluon has rapidity $y$ such that $0<y<Y / 2$, i.e., the gluon is emitted closer to onium 01 than to $0^{\prime} 1^{\prime}$ in the rapidity interval. The rapiditydependent gluon emissions of Fig. 2 generating small- $x$ evolution can be divided into three categories: (i) emission of "harder" gluons, with rapidity $\tilde{y}$ such that $y<\tilde{y}<Y / 2$; (ii) emission of "softer" gluons in the same onium's (01) wave function, i.e., emissions in the rapidity interval $0<$ $\tilde{y}<y$; (iii) emissions in the wave function of onium $0^{\prime} 1^{\prime}$, in the rapidity interval $-Y / 2<\tilde{y}<0$.

The analysis of category (i) and (ii) emissions is identical to that for gluon production in DIS carried out in Secs. III A and III B of [21] correspondingly and heavily relies on the real-virtual cancellations of the final state interactions discussed in [22]. In [21] the onium develops a dipole wave function which later interacts with the target nucleus. If the onium is moving along the light-cone "+" direction, the interval of the light-cone time $x_{+}$over which it interacts with the nucleus is negligibly short compared to the time it takes for the dipole wave function to develop $[10,35,36]$. This is very similar to onium-onium scattering: the light-cone interaction time between the two onia's wave functions is negligible compared to the time required to develop each of these wave functions. Thus, from the standpoint of one of these wave functions (the one in onium 01), the interaction is instantaneous, happening at, say, light-cone time $x_{+}=0$. Then, emissions of harder gluons [type (i)] may happen either before or after the interaction, at $x_{+}<0$ and $x_{+}>0$ correspondingly. They may be real (the gluon is present in the $x_{+}=+\infty$ final state) or virtual (the gluon is absent in the final state), given by Figs. 2 and 4 in [21] correspondingly. Because of the real-virtual cancellations of [22], the final state emissions/ absorptions of such gluons cancel, leaving only initial state radiation. The overall effect of type (i) emissions is to develop a dipole cascade before the collision which would lead to the creation of the "last" dipole in which the measured gluon is produced. In other words, instead of being emitted by the quark and antiquark in the original onium 01 , as shown in Fig. 4 leading to Eq. (15), the measured gluon would not be emitted in some dipole 23, generated by the dipole evolution.

The dipole number density at rapidity $y$ in the onium 01 is described by the quantity $n_{1}\left(\underline{x}_{01}, \underline{B}, Y / 2-\right.$ $\left.y ; \underline{x}_{23},(1 / 2)\left(\underline{x}_{2}+\underline{x}_{3}\right)\right)$ from Eq. (3) with $k=1$. Using Eq. (1) we see that it obeys the following differential equation [11,12], which is actually equivalent to the BFKL equation [9]:

$$
\begin{aligned}
\frac{\partial n_{1}\left(\underline{x}_{01}, \underline{B}, y ; \underline{x}_{23}, \underline{b}\right)}{\partial y}= & \frac{\alpha_{s} N_{c}}{2 \pi^{2}} \int d^{2} x_{2} \frac{x_{01}^{2}}{x_{20}^{2} x_{21}^{2}} \\
& \times\left[n_{1}\left(\underline{x}_{02}, \underline{B}+\frac{1}{2} \underline{x}_{21}, y ; \underline{x}_{23}, \underline{b}\right)\right. \\
& +n_{1}\left(\underline{x}_{12}, \underline{B}+\frac{1}{2} \underline{x}_{20}, y ; \underline{x}_{23}, \underline{b}\right) \\
& \left.-n_{1}\left(\underline{x}_{01}, \underline{B}, y ; \underline{x}_{23}, \underline{b}\right)\right]
\end{aligned}
$$

with the initial condition

$$
n_{1}\left(\underline{x}_{01}, \underline{B}, y=0 ; \underline{x}_{23}, \underline{b}\right)=\delta^{2}\left(\underline{x}_{01}-\underline{x}_{23}\right) \delta^{2}(\underline{B}-\underline{b}) \text {. }
$$

To include the effects of type (i) emissions one has to modify the cross section in Eq. (15) by [21,36]

$$
\begin{aligned}
\frac{d \sigma_{\mathrm{LO}}\left(\underline{x}_{01}, \underline{x}_{0^{\prime} 1^{\prime}}\right)}{d^{2} k d y d^{2} B} \rightarrow & \int d^{2} x_{23} d^{2} b n_{1}\left(\underline{x}_{01}, \underline{B}, Y / 2-y ; \underline{x}_{23}, \underline{b}\right) \\
& \times \frac{d \sigma_{\mathrm{LO}}\left(\underline{x}_{23}, \underline{x}_{0^{\prime} 1^{\prime}}\right)}{d^{2} k d y d^{2} b}
\end{aligned}
$$

where we are using the notations of [36], which are slightly different from those of [21]. 
The emissions of softer [type (ii)] gluons can again be analyzed similar to the DIS case of [21]. Analogous to how it was done in Sec. III B of [21], one can show that the effect of type (ii) emissions is to convert $d_{G q}$ in Eq. (15) into the gluon dipole/quark dipole $\left(0^{\prime} 1^{\prime}\right)$ forward scattering amplitude with a fully evolved wave function of the (upper) gluon dipole. However, to complete the picture one has to understand what happens to the wave function of the (lower) onium $0^{\prime} 1^{\prime}$, i.e., one has to analyze type (iii) emissions. This is rather straightforward: the lower onium $0^{\prime} 1^{\prime}$ develops a dipole wave function before the collision. In its light-cone time $x_{-}$the collision is, again, instantaneous, happening at $x_{-}=0$, after which it can again emit more gluons. However, none of the gluons emitted in this lower onium wave function is tagged upon, i.e., none of them is required to be present in the final state. Therefore, the rules for developing the lower onium wave functions are the same as for the calculation of the total onium-onium scattering cross sections: all final state emissions cancel [22], leaving only the initial state emissions shown in Fig. 2(a) and described by the generating functional $Z$ from Eq. (1). [We note also that there is no "cross talk"
( $s$-channel mergers) between emissions in the upper and lower onia in the leading logarithmic approximation considered here.] Combining what we know now about types (ii) and (iii) gluon emissions we conclude that they modify $d_{G q}$ in Eq. (15) into the gluon dipole/quark dipole forward scattering amplitude with fully evolved wave functions of both gluon and quark dipoles. To include types (ii) and (iii) gluon emissions into Eq. (15) we thus have to replace

$$
d_{G q}\left(\underline{r}, \underline{r}^{\prime}, \underline{b}\right) \rightarrow D_{G q}\left(\underline{r}, \underline{r}^{\prime}, \underline{b}, y+\frac{Y}{2}\right)
$$

in it. The quantity $D_{G q}$ can be defined by the analogy with $D$ from Eq. (7) by noting that, in the large- $N_{c}$ limit, the gluon dipole is equivalent to two quark dipoles, such that the corresponding generating functional is just a square of the generating functional for the quark dipole,

$$
Z_{G}\left(\underline{x}_{01}, \underline{B}, Y / 2, u\right)=\left[Z\left(\underline{x}_{01}, \underline{B}, Y / 2, u\right)\right]^{2},
$$

so that

$$
\begin{aligned}
D_{G q}\left(\underline{x}_{01}, \underline{x}_{0^{\prime} 1^{\prime}}, \underline{B}, Y\right)= & -\left\{\operatorname { e x p } \left[-\int d^{2} r d^{2} b d^{2} r^{\prime} d^{2} b^{\prime} d\left(\underline{r}, \underline{r}^{\prime}, \underline{b}-\underline{b}^{\prime}\right)\right.\right. \\
& \left.\left.\times \frac{\delta^{2}}{\delta u(\underline{r}, \underline{b}) \delta v\left(\underline{r}^{\prime}, \underline{b}^{\prime}\right)}\right] Z\left(\underline{x}_{01}, \underline{B}, Y / 2, u\right) Z\left(\underline{x}_{01}, \underline{B}, Y / 2, u\right) Z\left(\underline{x}_{0^{\prime} 1^{\prime}}, \underline{0},-Y / 2, v\right)\right\}\left.\right|_{u=1, v=1} .
\end{aligned}
$$

Combining Eqs. (19) and (20) we finally write for the inclusive gluon production cross section in onium-onium collisions

$$
\begin{aligned}
\frac{d \sigma\left(\underline{x}_{01}, \underline{x}_{0^{\prime} 1^{\prime}}\right)}{d^{2} k d y d^{2} B}= & \int d^{2} x_{2} d^{2} x_{3} n_{1}\left(\underline{x}_{01}, \underline{B}, \frac{Y}{2}-y ; \underline{x}_{23}, \frac{1}{2}\left(\underline{x}_{2}+\underline{x}_{3}\right)\right) \frac{\alpha_{s} C_{F}}{\pi^{2}} \frac{1}{(2 \pi)^{2}} \int d^{2} z_{1} d^{2} z_{2} e^{-i \underline{k} \cdot\left(\underline{z}_{1}-\underline{z}_{2}\right)} \sum_{i, j=2}^{3}(-1)^{i+j} \frac{\underline{z}_{1}-\underline{x}_{i}}{\left|\underline{z}_{1}-\underline{x}_{i}\right|^{2}} \\
& \times \frac{\underline{z}_{2}-\underline{x}_{j}}{\left|\underline{z}_{2}-\underline{x}_{j}\right|^{2}}\left[D_{G q}\left(\underline{z}_{1}-\underline{x}_{j}, \underline{x}_{0^{\prime} 1^{\prime}}, \frac{1}{2}\left(\underline{z}_{1}+\underline{x}_{j}\right), y+\frac{Y}{2}\right)+D_{G q}\left(\underline{z}_{2}-\underline{x}_{i}, \underline{x}_{0^{\prime} 1^{\prime}}, \frac{1}{2}\left(\underline{z}_{2}+\underline{x}_{i}\right), y+\frac{Y}{2}\right)\right. \\
& \left.-D_{G q}\left(\underline{z}_{1}-\underline{z}_{2}, \underline{x}_{0^{\prime} 1^{\prime}}, \frac{1}{2}\left(\underline{z}_{1}+\underline{z}_{2}\right), y+\frac{Y}{2}\right)-D_{G q}\left(\underline{x}_{i}-\underline{x}_{j}, \underline{x}_{0^{\prime} 1^{\prime}}, \frac{1}{2}\left(\underline{x}_{i}+\underline{x}_{j}\right), y+\frac{Y}{2}\right)\right]
\end{aligned}
$$

This is our main result. The above formula is valid for $0<$ $y<Y / 2$, and can be easily modified to give the production cross section for $-Y / 2<y<0$ by switching

$$
y \leftrightarrow-y, \quad \underline{x}_{01} \leftrightarrow \underline{x}_{0^{\prime} 1^{\prime}}
$$

If $y=0$ (the gluon is produced at midrapidity) Eq. (23) becomes symmetric, since $D_{g q}$ should contain only the linear BFKL evolution, due to the fact that the approximation to onium-onium scattering employed here $[1,11]$ does not consistently resum pomeron loops with a rapidity extent less or equal to $Y / 2$ (see Fig. 5 below). While formally Eq. (23) with $y=0$ sums up some pomeron loops of rapidity size $Y / 2$ and smaller in $D_{G q}$, as given by Eq. (22), such loops were not consistently summed over in arriving at Eq. (23). Therefore, all but the linear BFKL pomeron terms in $D_{G q}$ should be neglected in Eq. (23) at $y=0$.

It is interesting to notice that the evolution between the produced gluon and the closest onium [onium 01 in Eq. (23)] is given by the linear BFKL equation. This result is in accordance with the AGK cutting rules [23]. This is indeed surprising, particularly due to the fact that AGK violations have been found recently in the inclusive production of $q \bar{q}$ pairs [37] and in inclusive two-gluon production [38] in DIS and $p A$ collisions (see [26] for a relevant discussion). However, the reason our result (23) adheres to AGK rules is the same as for the single inclusive gluon production cross section in DIS: due to the nature of the approximation to the onium-onium scattering which we employed here following [1,11], there is no difference between the wave function of the onium closest to the 
produced gluon in rapidity and the dipole wave function of the $q \bar{q}$ pair in DIS, considered in [21]. Both wave functions include only pomeron splittings. This leads to the similarity between Eq. (23) and Eq. (30) in [21] and to the fact that both cross sections adhere to AGK cutting rules. While we cannot explain why some inclusive cross sections [21] adhere to AGK rules and some other cross sections $[37,38]$ violate them, it is clear that the reason AGK rules work for onium-onium scattering as well as for DIS is due to moderately high energy approximations applied with the nonlinear saturation effects being included only in one of the scatterers wave functions.

Similar to the result of [21], Eq. (23) can be recast in the $k_{T}$-factorization form [24]. First we note that, as long as impact parameters are concerned, $n_{1}(\underline{x}, \underline{B}, Y ; \underline{r}, \underline{b})$ depends only on $\underline{B}-\underline{b}$. Therefore,

$$
\begin{aligned}
& \int d^{2} B n_{1}\left(\underline{x}_{01}, \underline{B}, \frac{Y}{2}-y ; \underline{x}_{23}, \frac{1}{2}\left(\underline{x}_{2}+\underline{x}_{3}\right)\right) \\
& \equiv n_{1}\left(\underline{x}_{01}, \frac{Y}{2}-y ; \underline{x}_{23}\right)
\end{aligned}
$$

in Eq. (23) depends on $\underline{x}_{23}=\underline{x}_{2}-\underline{x}_{3}$, but does not depend on $\underline{x}_{2}+\underline{x}_{3}$. Using this fact we can recast Eq. (23) into the following form, similar to how it was done for Eq. (30) in [21] (note that now we do not assume that the amplitude $D_{G q}$ is independent of the impact parameter, as we did in [21]):

$$
\begin{aligned}
\frac{d \sigma\left(\underline{x}_{01}, \underline{x}_{0^{\prime} 1^{\prime}}\right)}{d^{2} k d y}= & \frac{\alpha_{s} C_{F}}{(2 \pi)^{3}} \frac{1}{\underline{k}^{2}} \int d^{2} z\left[\nabla_{z}^{2} \int d^{2} b D_{G q}\left(\underline{z}, \underline{x}_{0^{\prime} 1^{\prime}}, \underline{b}, y+\frac{Y}{2}\right)\right] e^{-i \underline{k} \underline{z}}\left[\nabla_{z}^{2} \sum_{i, j=2}^{3}(-1)^{i+j} \int d^{2} x_{23}\left(\underline{z}-\underline{x}_{i j}\right)^{2}\right. \\
& \left.\times \ln \left(\frac{1}{\left|\underline{z}-\underline{x}_{i j}\right| \mu}\right) n_{1}\left(\underline{x}_{01}, \frac{Y}{2}-y ; \underline{x}_{23}\right)\right] .
\end{aligned}
$$

For the derivation of Eq. (26) see the Appendix. Defining the unintegrated gluon distribution functions in the onia by

$$
\begin{aligned}
\phi_{01}(\underline{k}, y)= & \frac{C_{F}}{\alpha_{s}(2 \pi)^{3}} \int d^{2} z e^{-i \underline{k} \cdot \underline{z}} \nabla_{z}^{2}\left[\pi \alpha_{s}^{2} \sum_{i, j=2}^{3}(-1)^{i+j}\right. \\
& \times \int d^{2} x_{23}\left(\underline{z}-\underline{x}_{i j}\right)^{2} \ln \left(\frac{1}{\left|\underline{z}-\underline{x}_{i j}\right| \mu}\right) \\
& \left.\times n_{1}\left(\underline{x}_{01}, y ; \underline{x}_{23}\right)\right]
\end{aligned}
$$

and

$$
\phi_{0^{\prime} 1^{\prime}}(\underline{k}, y)=\frac{C_{F}}{\alpha_{s}(2 \pi)^{3}} \int d^{2} z d^{2} b e^{-i \underline{k} \cdot \underline{z}} \nabla_{z}^{2} D_{G q}\left(\underline{z}, \underline{x}_{0^{\prime} 1^{\prime}}, \underline{b}, y\right),
$$

we can rewrite Eq. (26) in the familiar $k_{T}$-factorized form $[21,24,25]$

$$
\begin{aligned}
\frac{d \sigma\left(\underline{x}_{01}, \underline{x}_{0^{\prime} 1^{\prime}}\right)}{d^{2} k d y}= & \frac{2 \alpha_{s}}{C_{F}} \frac{1}{\underline{k}^{2}} \int d^{2} q \phi_{01}\left(\underline{q}, \frac{Y}{2}-y\right) \\
& \times \phi_{0^{\prime} 1^{\prime}}\left(\underline{k}-\underline{q}, y+\frac{Y}{2}\right) .
\end{aligned}
$$

We have thus shown that the inclusive gluon production cross section in onium-onium scattering analyzed in the approximation of $[1,11]$ is given by the $k_{T}$-factorization formula (29).

\section{LIMITS OF APPLICABILITY OF THE POMERON LOOP RESUMMATION}

Equation (29) presents a problem. As long as $\phi_{01}$ and $\phi_{0^{\prime} 1^{\prime}}$ are positive definite, the inclusive cross section in Eq. (29) diverges as $\sim 1 / k^{2}$ in the infrared (at low $k_{T}=$ $|\underline{k}|)$. This is a well-known problem of perturbative gluon production [32-34] resulting in the infinite total number of gluons produced. The divergence is usually $\sim 1 / k_{T}^{4}$ at the lowest order (for $p p$ collisions) [32-34] shown in Fig. 4. It reduces to $\sim 1 / k_{T}^{2}$ for $p A$ collisions and DIS [35], when the saturation effects are included in the target nucleus. There is a strong belief in the community that including saturation effects both in the target and in the projectile wave functions would eliminate the power-law divergence completely. (Elimination of divergence would indeed require the $k_{T}$-factorization formula to break down, since no matter what the gluon distributions are it would always lead to $\sim 1 / k_{T}^{2}$ singularity in the IR.) The belief is confirmed by numerical [30,31] and analytic [29] analyses of gluon production in $A A$ collisions. Therefore, one would expect that a complete description of onium-onium scattering should also lead to an infrared-regular gluon production cross section. The fact that our result (29) does not give such a cross section indicates that the approach to oniumonium collisions from $[1,11]$ applied here is incomplete. (Indeed the same problem is present in DIS and $p A$ collisions in the approach used in $[17,21,35]$.)

To see what is missing in the approach of $[1,11]$ let us perform a few parametric estimates. First of all, a single BFKL pomeron exchange over rapidity interval $Y$ is, parametrically, of the order of

$$
\alpha_{s}^{2} e^{\left(\alpha_{P}-1\right) Y},
$$

where

$$
\alpha_{P}-1=\frac{4 \alpha_{s} N_{c}}{\pi} \ln 2
$$

is the intercept of the BFKL pomeron [9]. The exponent in Eq. (30) comes from a ladder rungs resummation with the factor of $\alpha_{s}^{2}$ in the traditional Feynman diagram language coming from the coupling of the ladder to the onia (impact 
factors). In the dipole model the factor $\alpha_{s}^{2}$ comes from the two-gluon exchange interaction between the two dipoles at the ends of the dipole evolutions in both onia [Eq. (4)].

Pomeron loops become important in onium-onium scattering at rapidity $Y_{U}$ at which the correction (30) brought in by an extra ladder becomes of the order of 1, i.e., when

$$
\alpha_{s}^{2} e^{\left(\alpha_{P}-1\right) Y_{U}} \sim 1
$$

leading to $[12,17]$

$$
Y_{U} \sim \frac{1}{\alpha_{P}-1} \ln \frac{1}{\alpha_{s}^{2}} .
$$

Therefore, for rapidities $Y \gtrsim Y_{U}$ pomeron loops become important and have to be resummed.

The types of pomeron loops included in the approach proposed in $[1,11]$ are shown in Fig. 5(a). There the thick lines denote BFKL ladders (hard pomerons). The approach of $[1,11]$ allows only for pomeron splittings at positive rapidities (above the dashed line) and only for pomeron mergers at negative rapidities (below the dashed line). Obviously this approximation consistently resums all pomeron loops stretching over a rapidity interval greater than $Y / 2$, where $Y$ is the total rapidity interval. However, some of the "smaller" pomeron loops, namely, the loops stretching over rapidity intervals less than $Y / 2$, are not included consistently. An example of pomeron loops which are not included is shown in Fig. 5(b), where the pomeron ladder both splits and merges at positive and at negative rapidities. One may argue that small pomeron loops are always subleading at high energies and can be safely neglected compared to larger loops. Still, the small loops of Fig. 5(b) become order 1 corrections at rapidity defined by

$$
\alpha_{S}^{2} e^{\left(\alpha_{P}-1\right) \tilde{Y}_{U} / 2} \sim 1,
$$

which gives

$$
\tilde{Y}_{U}=2 Y_{U}
$$

Therefore, we conclude that large pomeron loops of [1,11] are a good approximation to the high energy onium-onium scattering cross section only in the rapidity interval

$$
Y_{U} \leq Y<2 Y_{U} .
$$

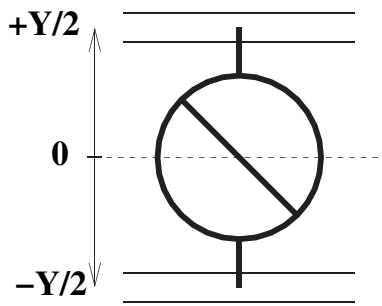

A

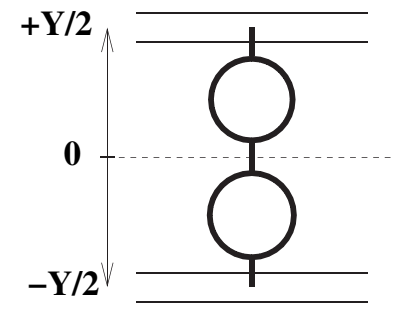

B
FIG. 5. The pomeron loop diagrams included (a) and not included (b) in the approach to onium-onium scattering of $[1,11]$. Thick lines denote BFKL ladders.
But what happens at higher rapidities, for $Y \geq 2 Y_{U}$ ? Would resummation of smaller pomeron loops of Fig. 5(b) be sufficient to consistently calculate the total onium-onium scattering cross section? Our answer is "no." The reason for this conclusion is that other (nonpomeron loop) higher order corrections also become important at $Y \geq 2 Y_{U}$. For instance, NLO corrections to the pomeron impact factor lead to a contribution of the order of [39]

$$
\alpha_{s}^{3} e^{\left(\alpha_{P}-1\right) Y}
$$

coming from the whole ladder. One may also speculate that inserting one rung of the NLO BFKL kernel [40] into the LO BFKL ladder [41] would generate the contribution of the same order as shown in Eq. (37). The corrections from Eq. (37) would become of order 1 at rapidity $(3 / 2) Y_{U}$. This would lead to an even smaller upper bound in Eq. (36), which would become $(3 / 2) Y_{U}$ instead of $2 Y_{U}$. The resulting applicability window for large pomeron loops is then limited to

$$
Y_{U} \leq Y<\frac{3}{2} Y_{U} .
$$

Indeed, since $Y_{U} \gg 1$ when $\alpha_{s} \ll 1$, the rapidity window in Eq. (38) would still be very large for asymptotically small coupling. Note that, for the values of the strong coupling constant accessible in the current accelerators, NLO BFKL corrections are numerically large and would modify our above conclusions.

At rapidity $Y=2 Y_{U}$ other corrections will become important as well. In performing the integration over rapidities of a leading order pomeron loop the lower limit of integration gives a loop stretching over the zero-rapidity interval $[13,17]$. Such a diagram would still have a single pomeron exchange, giving $e^{\left(\alpha_{P}-1\right) Y}$, and the zero size loop, giving $\alpha_{s}^{4}$ due to two pairs of dipoles interacting, overall contributing a factor of

$$
\alpha_{S}^{4} e^{\left(\alpha_{P}-1\right) Y} .
$$

A correction like the one shown in Eq. (39) may also result from inserting the NLO BFKL kernel [40] twice in the LO BFKL ladder [41], or from inserting the NNLO kernel once in the LO BFKL ladder.

The corrections in Eq. (39) become of order 1 when $Y=$ $2 Y_{U}$. In other words, these terms become comparable to small pomeron loops stretching over the $Y_{U}$ rapidity interval. This is natural since the resummation parameter for these higher order corrections is easily expressed in terms of the small pomeron loops resummation parameter

$$
\alpha_{S}^{4} e^{\left(\alpha_{P}-1\right) Y}=\left[\alpha_{S}^{2} e^{\left(\alpha_{P}-1\right) Y / 2}\right]^{2},
$$

so that both become comparable to 1 simultaneously.

We can conclude that pomeron loop approximation is valid only in the rapidity region specified by Eq. (38). To go beyond that rapidity interval one has to include a scope of other subleading nonpomeron loop effects listed above. 
The corrections of Eq. (39) were recently discussed in [6] to account for the apparent discrepancy between the results of $[2,5]$, indicating that such corrections cannot be consistently resummed within the current pomeron loop resummation formalisms.

Now let us return to the question of gluon production in onium-onium scattering. A typical diagram contributing to the above result (23) is shown in Fig. 6(a). There, the produced gluons are denoted by outgoing double arrows and the BFKL ladders are again denoted by thick lines. The graph in Fig. 6(a) has a linear BFKL evolution (single ladder) on one side of the produced gluon (in rapidity), with all the nonlinear pomeron loop effects being on the other side of the produced gluon, just like we have obtained in deriving Eq. (23). The resulting cross section is IR divergent as $\sim 1 / k_{T}^{2}$ and is valid in the rapidity window given by Eq. (38). Therefore, the $\sim 1 / k_{T}^{2}$ divergence persists as long as the pomeron loop approximation is valid.

Small pomeron loop corrections of Fig. 5(b) become important at rapidities $Y \geq 2 Y_{U}$. They may lead to gluon production diagrams like the one shown in Fig. 6(b). Such graphs include nonlinear saturation effects in the wave functions of both onia. However, they do not violate $k_{T}$ factorization, as can be seen in the graph of Fig. 6(b), which has no cross talk between the onia wave functions other then the interaction leading to gluon production. Therefore, diagrams like the one shown in Fig. 6(b) would still lead to $\sim 1 / k_{T}^{2}$ divergence in the infrared.

However, one may also imagine small pomeron loop corrections which would violate $k_{T}$ factorization, as shown in Fig. 6(c). As we discussed in Sec. III, in the large pomeron loop approximation of $[1,11]$ such corrections would cancel due to real-virtual cancellations of [22], making the final result for the inclusive cross section adhere to AGK cutting rules. Once the small pomeron loops of Fig. 6(b) are included in both of the onia wave functions, such real-virtual cancellations may stop being valid. This would lead to diagrams in Fig. 6(c) contributing to a gluon production cross section, and, possibly, regulating the $\sim 1 / k_{T}^{2}$ divergence. However, such regularization of the divergence would be due to small pomeron loops, which become important at rapidities $Y \geq 2 Y_{U}$, when the pomeron loop approximation breaks down. Thus it may happen that regularization of $\sim 1 / k_{T}^{2}$ divergence would have to also include effects going beyond the pomeron loop approximation, alongside the purely pomeron loop diagrams of Fig. 6(c).

If, instead of going to higher energies/rapidities, we fix the rapidity $Y$ and decrease $k_{T}$, "higher twist" effects would become progressively more important. Our conclusion above implies that, while higher twist terms due to large pomeron loops of $[1,11]$ do not regularize the $\sim 1 / k_{T}^{2}$ divergence of the inclusive gluon production cross section, the higher twist terms associated with small pomeron loops, not included in the approximation of $[1,11]$, which may regulate the $\sim 1 / k_{T}^{2}$ singularity, come in at the same time as the higher twist terms associated with other (nonpomeron loop) kinds of corrections.

\section{ACKNOWLEDGMENTS}

This work is supported in part by the U.S. Department of Energy under Grant No. DE-FG02-05ER41377.

\section{APPENDIX}

Starting with the first term in the square brackets of Eq. (23) (integrated over $\underline{B}$ ) we note that the $z_{2}$ integral can be carried out using

$$
\int d^{2} z e^{i k \underline{k} \underline{z}} \underline{\underline{z}} \underline{z}^{2}=2 \pi i \frac{k}{\underline{k}^{2}}
$$

yielding

$$
\begin{aligned}
\int d^{2} x_{2} & d^{2} x_{3} n_{1}\left(\underline{x}_{01}, \frac{Y}{2}-y ; \underline{x}_{23}\right) \\
& \times \frac{\alpha_{s} C_{F}}{2 \pi^{3}} i \int d^{2} z_{1} e^{\left.-i \underline{i} \cdot \underline{z}_{1}-\underline{x}_{j}\right)} \sum_{i, j=2}^{3}(-1)^{i+j} \frac{\underline{z}_{1}-\underline{x}_{i}}{\left|\underline{z}_{1}-\underline{x}_{i}\right|^{2}} \\
& \frac{\underline{k}}{\underline{k}^{2}} D_{G q}\left(\underline{z}_{1}-\underline{x}_{j}, \underline{x}_{0^{\prime}} 1^{\prime}, \frac{1}{2}\left(\underline{z}_{1}+\underline{x}_{j}\right), y+\frac{Y}{2}\right) .
\end{aligned}
$$

Defining $\underline{z}^{\prime}=\underline{z}_{1}-\underline{x}_{j}$ and rewriting $d^{2} x_{2} d^{2} x_{3}=d^{2} x_{23} d^{2} b$,

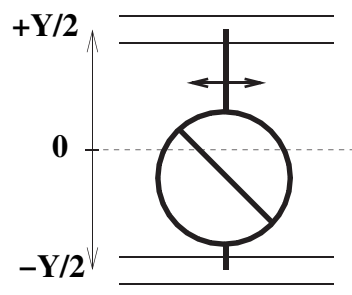

A

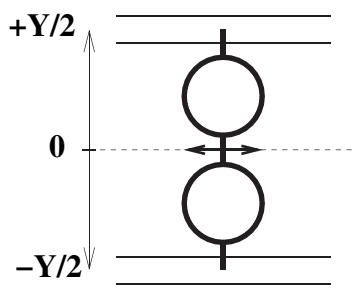

B

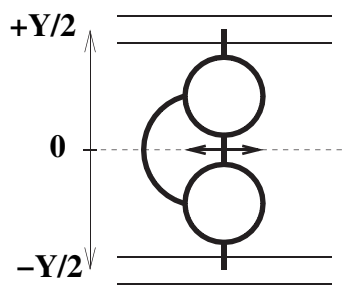

C

FIG. 6. Gluon production diagrams for onium-onium scattering: (a) a typical diagram contributing to Eq. (23); (b) contributions of smaller pomeron loops still giving an IR-singular gluon production cross section; (c) contributions of smaller pomeron loops which may lead to IR-regular gluon production cross section. Again thick lines denote BFKL ladders and outgoing arrows denote produced gluons. 
where $\underline{b}=\underline{x}_{j}+(1 / 2) \underline{z}$ we can transform Eq. (A2) into

$$
\begin{aligned}
& \int d^{2} x_{23} n_{1}\left(\underline{x}_{01}, \frac{Y}{2}-y ; \underline{x}_{23}\right) \\
& \quad \times \frac{\alpha_{s} C_{F}}{2 \pi^{3}} i \int d^{2} z e^{-i \underline{k} \cdot \underline{z}} \sum_{i, j=2}^{3}(-1)^{i+j} \frac{\underline{z}-\underline{x}_{i j}}{\left|\underline{z}-\underline{x}_{i j}\right|^{2}} \cdot \frac{\underline{k}}{\underline{k}^{2}} \\
& \quad \times \int d^{2} b D_{G q}\left(\underline{z}, \underline{x}_{0^{\prime} 1^{\prime}}, \underline{b}, y+\frac{Y}{2}\right) .
\end{aligned}
$$

The second term in the square brackets of Eq. (23) gives an identical contribution.

The third term in the square brackets of Eq. (23) is different. Defining $\tilde{z}_{1}=\underline{z}_{1}-\underline{x}_{i}$ and $\tilde{z}_{2}=\underline{z}_{2}-\underline{x}_{j}$ we write it as

$$
\begin{aligned}
-\int d^{2} & x_{2} d^{2} x_{3} n_{1}\left(\underline{x}_{01}, \frac{Y}{2}-y ; \underline{x}_{23}\right) \frac{\alpha_{s} C_{F}}{(2 \pi)^{2} \pi^{2}} \\
& \times \int d^{2} \tilde{z}_{1} d^{2} \tilde{z}_{2} \frac{\tilde{z}_{1}}{\tilde{z}_{1}^{2}} \cdot \frac{\tilde{z}_{2}}{\tilde{z}_{2}^{2}} \sum_{i, j=2}^{3}(-1)^{i+j} e^{-i \underline{k} \cdot\left(\tilde{z}_{1}-\tilde{z}_{2}+\underline{x}_{i j}\right)} \\
& \times D_{G q}\left(\tilde{z}_{1}-\tilde{z}_{2}+\underline{x}_{i j}, \underline{x}_{0^{\prime} 1^{\prime}}, \frac{1}{2}\left(\tilde{z}_{1}+\tilde{z}_{2}\right)\right. \\
& \left.+\frac{1}{2}\left(\underline{x}_{i}+\underline{x}_{j}\right), y+\frac{Y}{2}\right) .
\end{aligned}
$$

Dropping tildes over $\underline{z}_{1}$ and $\underline{z}_{2}$, rewriting $d^{2} x_{2} d^{2} x_{3}=$ $d^{2} x_{23} d^{2} b$ with

$$
\underline{b}=\frac{1}{2}\left(\underline{x}_{i}+\underline{x}_{j}\right)+\frac{1}{2}\left(\tilde{z}_{1}+\tilde{z}_{2}\right)
$$

we recast Eq. (A4) into

$$
\begin{gathered}
-\int d^{2} x_{23} n_{1}\left(\underline{x}_{01}, \frac{Y}{2}-y ; \underline{x}_{23}\right) \frac{\alpha_{s} C_{F}}{(2 \pi)^{2} \pi^{2}} \int d^{2} z_{1} d^{2} z_{2} \frac{\underline{z}_{1}}{\underline{z}_{1}^{2}} \cdot \frac{\underline{z}_{2}}{\underline{z}_{2}^{2}} \\
\times \sum_{i, j=2}^{3}(-1)^{i+j} e^{\left.-i \underline{k} \cdot \underline{z}_{1}-\underline{z}_{2}+\underline{x}_{i j}\right)} \\
\times \int d^{2} b D_{G q}\left(\underline{z}_{1}-\underline{z}_{2}+\underline{x}_{i j}, \underline{x}_{0^{\prime} 1^{\prime}}, \underline{b}, y+\frac{Y}{2}\right) .
\end{gathered}
$$

Defining $\underline{z}=\underline{z}_{1}-\underline{z}_{2}+\underline{x}_{i j}$ and integrating over $\underline{y}=$ $(1 / 2)\left(\underline{z}_{1}+\underline{z}_{2}\right)$ using

$$
\int d^{2} y \frac{y}{\underline{y}^{2}} \cdot \frac{\underline{y}+\underline{z}}{|\underline{y}+\underline{z}|^{2}}=2 \pi \ln \frac{1}{|\underline{z}| \mu}
$$

with $\mu$ some infrared cutoff yields

$$
\begin{aligned}
& -\int d^{2} x_{23} n_{1}\left(\underline{x}_{01}, \frac{Y}{2}-y ; \underline{x}_{23}\right) \frac{\alpha_{s} C_{F}}{(2 \pi) \pi^{2}} \\
& \times \int d^{2} z \sum_{i, j=2}^{3}(-1)^{i+j} e^{-i \underline{k} \cdot \underline{z}} \ln \left(\frac{1}{\left|\underline{z}-\underline{x}_{i j}\right| \mu}\right) \\
& \times \int d^{2} b D_{G q}\left(\underline{z}, \underline{x}_{0^{\prime} 1^{\prime}}, \underline{b}, y+\frac{Y}{2}\right) \text {. }
\end{aligned}
$$

Finally, similar to the above, the last term in the square brackets of Eq. (23) can be written as

$$
\begin{aligned}
& -\int d^{2} x_{23} n_{1}\left(\underline{x}_{01}, \frac{Y}{2}-y ; \underline{x}_{23}\right) \frac{\alpha_{s} C_{F}}{\pi^{2}} \frac{1}{\underline{k}^{2}} \\
& \times \int d^{2} z \sum_{i, j=2}^{3}(-1)^{i+j} e^{-i \underline{k} \cdot \underline{z}} \delta^{2}\left(\underline{z}-\underline{x}_{i j}\right) \\
& \quad \times \int d^{2} b D_{G q}\left(\underline{z}, \underline{x}_{0^{\prime} 1^{\prime}}, \underline{b}, y+\frac{Y}{2}\right) .
\end{aligned}
$$

Combining Eqs. (A3) (twice), (A7) and (A8) yield

$$
\begin{aligned}
\frac{d \sigma\left(\underline{x}_{01}, \underline{x}_{0^{\prime} 1^{\prime}}\right)}{d^{2} k d y}= & \int d^{2} x_{23} n_{1}\left(\underline{x}_{01}, \frac{Y}{2}-y ; \underline{x}_{23}\right) \frac{\alpha_{s} C_{F}}{2 \pi^{3}} \frac{1}{\underline{k}^{2}} \\
& \times \int d^{2} z \sum_{i, j=2}^{3}(-1)^{i+j} e^{-i \underline{k} \cdot \underline{z}} \\
& \times \int d^{2} b D_{G q}\left(\underline{z}, \underline{x}_{0^{\prime} 1^{1}}, \underline{b}, y+\frac{Y}{2}\right) \\
& \times\left[2 i \underline{k} \cdot \frac{\underline{z}-\underline{x}_{i j}}{\left|\underline{z}-\underline{x}_{i j}\right|^{2}}-\underline{k}^{2} \ln \left(\frac{1}{\left|\underline{z}-\underline{x}_{i j}\right| \mu}\right)\right. \\
& \left.-2 \pi \delta^{2}\left(\underline{z}-\underline{x}_{i j}\right)\right],
\end{aligned}
$$

which can be rewritten as

$$
\begin{aligned}
\frac{d \sigma\left(\underline{x}_{01}, \underline{x}_{0^{\prime} 1^{\prime}}\right)}{d^{2} k d y}= & \int d^{2} x_{23} n_{1}\left(\underline{x}_{01}, \frac{Y}{2}-y ; \underline{x}_{23}\right) \frac{\alpha_{s} C_{F}}{2 \pi^{3}} \frac{1}{\underline{k}^{2}} \\
& \times \int d^{2} z d^{2} b D_{G q}\left(\underline{z}, \underline{x}_{0^{\prime} 1^{\prime}}, \underline{b}, y+\frac{Y}{2}\right) \\
& \times \sum_{i, j=2}^{3}(-1)^{i+j} \nabla_{z}^{2}\left[e^{-i \underline{k} \cdot \underline{z}} \ln \left(\frac{1}{\left|\underline{z}-\underline{x}_{i j}\right| \mu}\right)\right],
\end{aligned}
$$

where $\nabla_{z}^{2}$ is the transverse coordinate (2D) gradient squared. Integrating by parts yields

$$
\begin{aligned}
\frac{d \sigma\left(\underline{x}_{01}, \underline{x}_{0^{\prime} 1^{\prime}}\right)}{d^{2} k d y}= & \frac{\alpha_{s} C_{F}}{2 \pi^{3}} \frac{1}{k^{2}} \int d^{2} z \\
& \times\left[\nabla_{z}^{2} \int d^{2} b D_{G q}\left(\underline{z}, \underline{x}_{0^{\prime} 1^{\prime}}, \underline{b}, y+\frac{Y}{2}\right)\right] \\
& \times e^{-i \underline{k} \cdot \underline{z}} \sum_{i, j=2}^{3}(-1)^{i+j} \int d^{2} x_{23} \\
& \times n_{1}\left(\underline{x}_{01}, \frac{Y}{2}-y ; \underline{x}_{23}\right) \ln \left(\frac{1}{\left|\underline{z}-\underline{x}_{i j}\right| \mu}\right)
\end{aligned}
$$

and, remembering that

$$
\nabla_{z}^{2}\left(\underline{z}^{2} \ln \frac{1}{|\underline{z}| \mu}\right)=4 \ln \frac{1}{|\underline{z}| \mu}
$$

reduces Eq. (A11) to Eq. (26) as desired. 
[1] E. Iancu and A. H. Mueller, Nucl. Phys. A730, 460 (2004); A730, 494 (2004).

[2] A. H. Mueller and A. I. Shoshi, Nucl. Phys. B692, 175 (2004); A. H. Mueller, A. I. Shoshi, and S. M. H. Wong, Nucl. Phys. B715, 440 (2005).

[3] E. Iancu and D. N. Triantafyllopoulos, Nucl. Phys. A756, 419 (2005); Phys. Lett. B 610, 253 (2005); J. P. Blaizot, E. Iancu, K. Itakura, and D. N. Triantafyllopoulos, Phys. Lett. B 615, 221 (2005); Y. Hatta, E. Iancu, L. McLerran, A. Stasto, and D. N. Triantafyllopoulos, hep-ph/0504182.

[4] E. Levin and M. Lublinsky, Phys. Lett. B 607, 131 (2005); hep-ph/0501173.

[5] A. Kovner and M. Lublinsky, Phys. Rev. D 71, 085004 (2005); J. High Energy Phys. 03 (2005) 001; Phys. Rev. Lett. 94, 181603 (2005); Phys. Rev. D 72, 074023 (2005).

[6] C. Marquet, A. H. Mueller, A. I. Shoshi, and S.M.H. Wong, hep-ph/0505229.

[7] I. Balitsky, hep-ph/0507237 [Phys. Rev. D (to be published)].

[8] M. Kozlov and E. Levin, Nucl. Phys. A739, 291 (2004); S. Bondarenko, M. Kozlov, and E. Levin, Acta Phys. Pol. B 34, 3081 (2003); M. Kozlov and E. Levin, Eur. Phys. J. C 28, 483 (2003).

[9] E. A. Kuraev, L. N. Lipatov, and V.S. Fadin, Zh. Eksp. Teor. Fiz. 72, 377 (1977) [Sov. Phys. JETP 45, 199 (1977)]; I. I. Balitsky and L. N. Lipatov, Yad. Fiz. 28, 1597 (1978) [Sov. J. Nucl. Phys. 28, 822 (1978)].

[10] A. H. Mueller, Nucl. Phys. B415, 373 (1994).

[11] A. H. Mueller and B. Patel, Nucl. Phys. B425, 471 (1994).

[12] A. H. Mueller, Nucl. Phys. B437, 107 (1995).

[13] H. Navelet and R. Peschanski, Phys. Rev. Lett. 82, 1370 (1999).

[14] G. P. Salam, Nucl. Phys. B461, 512 (1996); A. H. Mueller and G. P. Salam, Nucl. Phys. B475, 293 (1996).

[15] L. V. Gribov, E. M. Levin, and M. G. Ryskin, Phys. Rep. 100, 1 (1983); A. H. Mueller and J.w. Qiu, Nucl. Phys. B268, 427 (1986).

[16] L. D. McLerran and R. Venugopalan, Phys. Rev. D 49, 2233 (1994); 49, 3352 (1994); 50, 2225 (1994).

[17] Y. V. Kovchegov, Phys. Rev. D 60, 034008 (1999); 61, 074018 (2000).

[18] I. Balitsky, Nucl. Phys. B463, 99 (1996); hep-ph/9706411; Phys. Rev. D 60, 014020 (1999).

[19] J. Jalilian-Marian, A. Kovner, A. Leonidov, and H. Weigert, Nucl. Phys. B504, 415 (1997); Phys. Rev. D 59, 014014 (1999); 59, 034007 (1999); 59, 099903(E) (1999); 59, 014015 (1999); A. Kovner, J. G. Milhano, and H. Weigert, Phys. Rev. D 62, 114005 (2000); H.
Weigert, Nucl. Phys. A703, 823 (2002).

[20] E. Iancu, A. Leonidov, and L. D. McLerran, Nucl. Phys. A692, 583 (2001); Phys. Lett. B 510, 133 (2001); E. Iancu and L. D. McLerran, Phys. Lett. B 510, 145 (2001); E. Ferreiro, E. Iancu, A. Leonidov, and L. McLerran, Nucl. Phys. A703, 489 (2002).

[21] Yu. V. Kovchegov and K. Tuchin, Phys. Rev. D 65, 074026 (2002).

[22] Z. Chen and A. H. Mueller, Nucl. Phys. B451, 579 (1995).

[23] V. A. Abramovsky, V. N. Gribov, and O. V. Kancheli, Yad. Fiz. 18, 595 (1973) [Sov. J. Nucl. Phys. 18, 308 (1974)].

[24] E. M. Levin and M. G. Ryskin, Yad. Fiz. 21, 1072 (1975).

[25] M. A. Braun, Phys. Lett. B 483, 105 (2000).

[26] M. A. Braun, Eur. Phys. J. C 39, 451 (2005); 42, 169 (2005).

[27] C. Marquet, Nucl. Phys. B705, 319 (2005).

[28] M. Ciafaloni, Nucl. Phys. B296, 49 (1988); S. Catani, F. Fiorani, and G. Marchesini, Phys. Lett. B 234, 339 (1990); Nucl. Phys. B336, 18 (1990); G. Marchesini, Nucl. Phys. B445, 49 (1995).

[29] Y. V. Kovchegov, Nucl. Phys. A692, 557 (2001).

[30] A. Krasnitz and R. Venugopalan, Phys. Rev. Lett. 84, 4309 (2000); 86, 1717 (2001); A. Krasnitz, Y. Nara, and R. Venugopalan, Phys. Rev. Lett. 87, 192302 (2001); Nucl. Phys. A727, 427 (2003); A717, 268 (2003).

[31] T. Lappi, Phys. Rev. C 67, 054903 (2003).

[32] A. Kovner, L. D. McLerran, and H. Weigert, Phys. Rev. D 52, 3809 (1995); 52, 6231 (1995).

[33] Yu. V. Kovchegov and D. H. Rischke, Phys. Rev. C 56, 1084 (1997).

[34] M. Gyulassy and L. D. McLerran, Phys. Rev. C 56, 2219 (1997).

[35] Y. V. Kovchegov and A. H. Mueller, Nucl. Phys. B529, 451 (1998).

[36] J. Jalilian-Marian and Y. V. Kovchegov, hep-ph/0505052.

[37] J.P. Blaizot, F. Gelis, and R. Venugopalan, Nucl. Phys. A743, 57 (2004); A743, 13 (2004); F. Gelis and R. Venugopalan, Phys. Rev. D 69, 014019 (2004).

[38] J. Jalilian-Marian and Y. V. Kovchegov, Phys. Rev. D 70, 114017 (2004); 71, 079901 (2005).

[39] J. Bartels, D. Colferai, S. Gieseke, and A. Kyrieleis, Phys. Rev. D 66, 094017 (2002); J. Bartels, S. Gieseke, and A. Kyrieleis, Phys. Rev. D 65, 014006 (2002).

[40] V.S. Fadin and L. N. Lipatov, Phys. Lett. B 429, 127 (1998); M. Ciafaloni and G. Camici, Phys. Lett. B 430, 349 (1998).

[41] Y. V. Kovchegov and A. H. Mueller, Phys. Lett. B 439, 428 (1998). 